Research Article

\title{
A Theoretical Investigation of Channel Wave Multipath Propagation in a Coal Seam
}

\author{
Yinjing Guo $\mathbb{D}$, Jianhua Zhang $(\mathbb{D}$, Yuanyuan Ju $\mathbb{D}$, and Xiaohan Guo \\ College of Electronic and Information Engineering, Shandong University of Science and Technology, Qingdao 266590, China \\ Correspondence should be addressed to Yinjing Guo; gyjlwh@163.com
}

Received 11 June 2019; Revised 30 July 2019; Accepted 5 September 2019; Published 3 November 2019

Academic Editor: Gareth A. Vio

Copyright (C) 2019 Yinjing Guo et al. This is an open access article distributed under the Creative Commons Attribution License, which permits unrestricted use, distribution, and reproduction in any medium, provided the original work is properly cited.

\begin{abstract}
This study investigates the mechanism of channel wave multipath propagation to determine features of a channel wave in a coal seam. For this purpose, deduction formulas were used for different time delays and propagation paths and, subsequently, a multipath fading channel wave pattern was built. MATLAB software was used to simulate the model. To this end, the characteristics of the coal seam and the surrounding rock were considered. The simulation results were compared with results obtained from the model contained in COMSOL software to verify the accuracy of the model. According to the results obtained from the simulation, the proposed model shows a good match with the COMSOL model, which is a common simulation comparison standard. In addition, the channel wave energy approximates to exponential decay at a constant distance from the source, and the multipath propagation considerably affects its energy attenuation. Furthermore, the multipath reflections' travel time derivation is accomplished properly in the coal seam.
\end{abstract}

\section{Introduction}

When using an elastic wave to probe a coal seam, a specific elastic wave is connected to the coal seam using a transmitter $[1,2]$. According to the weak elastic wave signal detected by the receiver, the detection system records the geological structure of the coal seam through signal processing and analysis $[3,4]$. When elastic waves propagate in the formation, the elastic wave reflection [5], refraction [6], and transmission [7] characteristics make the composition of the elastic wave very complicated, resulting in a multipath effect [8]. Under the influence of this effect, the signal is transmitted via various paths to reach the receiving end. Multipath propagation creates two problems: fading and dispersion $[9,10]$. The various propagation velocities that form the frequency of the elastic waves cause dispersion. Meanwhile, channel wave losses in the formation cause fading [11]. In particular, the multipath fading caused by signal reflection, which may vary greatly [12], may change the transmitted signal. There are many studies on the multipath propagation phenomenon of electromagnetic waves at home and abroad [13-15], but there are few studies on the multipath effect of elastic waves in a geological formation. Zhang et al. established the attenuation and time delay model of a multipath channel by analyzing the attenuation and delay characteristics of multipath propagation in a tunnel [16]. Hao et al. obtained a multipath channel model for elastic wave signal propagation in a homogeneous medium by analyzing the attenuation characteristics of elastic waves propagating in parallel layers [17]. Gao and Huang observed that the multipath effect on elastic waves is determined by the characteristics of the earth channel during transmission and is the main factor affecting the performance of a through-the-earth communication system [18].

The main contribution of this paper is to try to explore the signal fading problem in the fine detection of coal channel transmission trough wave. The characteristics of elastic wave multipath fading are analyzed based on the multipath effect which has great influence on fading. A new multipath propagation model is established, and the multipath propagation of elastic waves is simulated by MATLAB and COMSOL. The correctness of the model was verified. Through the work of this paper, it can provide theoretical basis and fundamental guarantee for the study of the elastic 
wave receiving method of formation propagation. Meanwhile, it provides a new method for detecting stratum structure during coal mining, which is of great significance for coal seam exploration.

This paper is divided into four parts. This first part discusses the role and characteristics of elastic waves and the research status of multipath effects on elastic waves propagating in a formation. The second part discusses the mechanism of elastic wave multipath propagation, the path difference of multipath propagation and time delay, and the energy attenuation of multipath signals. In the third part, based on the established multipath propagation model of elastic waves in a coal seam, the propagation of elastic waves in a coal seam is simulated. Finally, our conclusions are summarized in the fourth part.

\section{Model of Channel Wave Multipath Propagation in a Coal Seam}

2.1. Mechanism of Elastic Wave Multipath Propagation. In the current study, the coal seam is assumed to be a homogeneous medium that is in parallel to the rock around it. A coal seam channel wave multipath propagation model is developed in which the diffraction propagation of the surrounding rock is ignored. It is assumed that the receiver detection sensor $R$ and the transmitter transducer $T$ are positioned in the way indicated in Figure 1. The height of the coal seam is denoted by $H$, and the heights of $T$ and $R$ from the bottom of the seam are both H/2. $d$ denotes the transmitter-receiver interval. In the first situation, waves of the channel are reflected onto the lower interface. This situation is considered first as when waves reflect at both the higher and lower interfaces, the same interaction occurs.

2.1.1. The Collineation Path. T launches the signal, and they are received by $R$ though the path $L$; this is shown as the direct path signal. The $L$ value is expressed as follows:

$$
L=d \text {. }
$$

2.1.2. The Initial Reflection Path. T launches the signal, and they arrive at $P_{11}$ by $L_{11}$. Some signals travel into the surrounding rock (this type of signal is not considered here), while other signals are reflected by $P_{11}$, and they are received by $R$ through $L_{12}$. The total path $L_{1}$ for these reflection signals is expressed as follows:

$$
L_{1}=L_{11}+L_{12}=\sqrt{\left(\frac{H}{2}+\frac{H}{2}\right)^{2}+d^{2}}=\sqrt{H^{2}+d^{2}} .
$$

$\theta_{1}$ is the incidence angle of this signal. Hence,

$$
\sin \theta_{1}=\frac{d}{L_{11}+L_{12}}=\frac{d}{\sqrt{H^{2}+d^{2}}} .
$$

2.1.3. The Secondary Reflection Path. T sends the signal, and it arrives at $P_{21}$ by $L_{21}$. Some signals are transferred into the

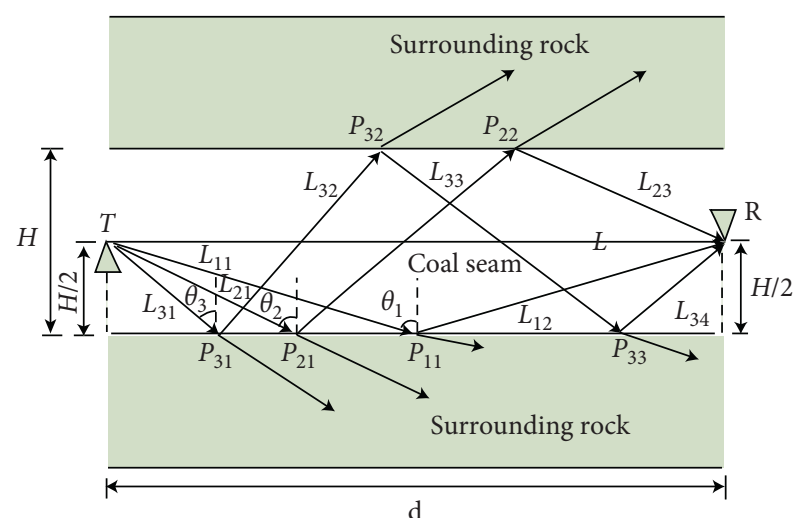

FIgURE 1: The diagram of a multipath signal that reflects the higher and lower interfaces.

enclosing rock, while other signals reach $P_{22}$ by $L_{22}$. A part of the latter signals is transferred into the surrounding rock, and $R$ receives the remaining part. This signal is referred to as the secondary reflection signal, and the total path $L_{2}$ is expressed as follows:

$$
\begin{aligned}
L_{2} & =L_{21}+L_{22}+L_{23}=\sqrt{\left(\frac{H}{2}+H+H-\frac{H}{2}\right)^{2}+d^{2}} \\
& =\sqrt{(2 H)^{2}+d^{2}} .
\end{aligned}
$$

$\theta_{2}$ represents this signal's incidence angle. Hence,

$$
\sin \theta_{2}=\frac{d}{L_{21}+L_{22}+L_{23}}=\frac{d}{\sqrt{(2 H)^{2}+d^{2}}} .
$$

2.1.4. The Triple Reflection Path. T sends the signal, and it arrives at $P_{31}$ by $L_{31}$. Some signals are transferred into the enclosing rock, while other signals reach $P_{32}$ by $L_{32}$. A part of the latter signals is transferred into the enclosing rock, and the remaining part reaches $P_{33}$ by $L_{33}$. In addition, one part of the latter signals is partially transferred into the rock, whereas $R$ receives the other part through $L_{34}$. This signal is referred to as the triple reflection signal total path, of which $L_{2}$ is expressed as follows:

$$
\begin{aligned}
L_{3} & =L_{31}+L_{32}+L_{33}+L_{34}=\sqrt{\left(\frac{H}{2}+2 H+\frac{H}{2}\right)^{2}+d^{2}} \\
& =\sqrt{(3 H)^{2}+d^{2}} .
\end{aligned}
$$

$\theta_{3}$ represents the signal's incidence angle. Hence,

$$
\sin \theta_{3}=\frac{d}{L_{31}+L_{32}+L_{33}+L_{34}}=\frac{d}{\sqrt{(3 H)^{2}+d^{2}}} .
$$

This process continues in the same way.

The assumed coal seam contains a gentle and flat interface parallel to the enclosing rock. Therefore, when scattering and reflection are not considered in its microstructure, the following process is used [16]. 
The received signal includes a signal reflected from the bottom and top of the coal seam, and a direct path signal. It is assumed that the coal seam includes $Z$ paths (each reflected signal has one path), and $N$ denotes the number of received signals. Hence, $N=1+2 Z$.

For a path with multiple reflections (the first originating from the interface) in a coal seam, it is assumed that $n(n=1$, $2,3, \ldots)$ denotes the number of reflections. Thus, the path includes $n+1$ broken lines, and the following relation expresses the total path length:

$$
\begin{aligned}
L_{n} & =L_{n 1}+L_{n 2}+\cdots+L_{n(n+1)} \\
& =\sqrt{\left[\frac{H}{2}+(n-1) H+H-\frac{H}{2}\right]^{2}+d^{2}}=\sqrt{(n H)^{2}+d^{2}},
\end{aligned}
$$

where $H$ denotes the height of the coal seam. $d$ denotes the transmitter-receiver interval. $\theta_{n}$ represents the assumed incident angle of the $n$th reflected signal. Hence,

$$
\sin \theta_{n}=\frac{d}{L_{n 1}+L_{n 2}+\cdots+L_{n(n+1)}}=\frac{d}{\sqrt{(n H)^{2}+d^{2}}} .
$$

\subsection{Path Difference and Time Delay of Multipath} Propagation. Path difference occurs among multipath signals at the time of propagation of channel waves in a coal seam, causing signal delay. Multipath fading develops as a result of the multipath propagation of channel waves at the time of reception of the signal by the receiver. This can have a significant impact on the received signal's quality [19-21]. It is possible to deduce the path difference between the multipath signal and the direct signal, along with the time delay formula in the multipath signal, by considering the direct wave based on the path formula and multipath propagation model given above.

$\Delta d_{1}$ denotes the difference between the primary reflection path and the direct path difference at the bottom of the coal seam, expressed as follows:

$$
\Delta d_{1}=L_{1}-L=\sqrt{H^{2}+d^{2}}-d .
$$

$\Delta t_{1}$ denotes the one-time reflection signal propagation that is expressed as follows:

$$
\Delta t_{1}=\frac{\Delta d_{1}}{v}=\frac{L_{1}-L}{v}=\frac{\sqrt{H^{2}+d^{2}}-d}{v} .
$$

$v$ denotes the channel waves' propagation velocity in the coal seam. The height is equal to $H / 2$ for both the transceivers. It means that there is a relationship between propagation delay and transmitter-receiver distance, and it is not dependent on the initial radiation direction at the height of the transceivers.

$\Delta d_{2}$ represents the path difference between the secondary reflection path and the direct path difference at the bottom of the coal seam, expressed as follows:

$$
\Delta d_{2}=L_{2}-L=\sqrt{(2 H)^{2}+d^{2}}-d .
$$

$\Delta t_{2}$ denotes the second reflection signal propagation delay that is expressed as follows:

$$
\Delta t_{2}=\frac{\Delta d_{2}}{v}=\frac{\sqrt{(2 H)^{2}+d^{2}}-d}{v} .
$$

The equation given above indicates that there is a close relationship between the time delay, the transmitter-receiver distance, and the thickness of the coal seam. Therefore, it is not dependent on the initial departure angle.

$\Delta d_{3}$ denotes the path difference between the third reflection path and the direct path difference at the bottom of the coal seam:

$$
\Delta d_{3}=L_{3}-L=\sqrt{(3 H)^{2}+d^{2}}-d .
$$

$\Delta t_{3}$ denotes the third reflection signal propagation delay that is expressed as follows:

$$
\Delta t_{3}=\frac{\Delta d_{3}}{v}=\frac{\sqrt{(3 H)^{2}+d^{2}}-d}{v} .
$$

According to the mechanism of the multipath propagation, the path difference between the $n$th reflection $L_{n}$ path and the direct path difference $L$ at the bottom of the coal seam is $\Delta d_{n}(n=1,2,3, \ldots)$, expressed as follows:

$$
\begin{aligned}
\Delta d_{n} & =L_{n}-L=\sqrt{\left[\frac{H}{2}+(n-1) H+\left(H-\frac{H}{2}\right)\right]^{2}+d^{2}}-d \\
& =\sqrt{(n H)^{2}+d^{2}}-d .
\end{aligned}
$$

The following equation gives the $n$th propagation delay between the reflected path and the direct path:

$$
\begin{aligned}
\Delta t_{n} & =\frac{\Delta d_{n}}{v}=\frac{\sqrt{[(H / 2)+(n-1) H+(H-(H / 2))]^{2}+d^{2}}-d}{v} \\
& =\frac{\sqrt{(n H)^{2}+d^{2}}-d}{v},
\end{aligned}
$$

where $n=1,2,3, \ldots$ and $v$ denotes the channel wave propagation velocity in the coal seam.

Considering equation (17), if the height from the bottom of the coal seam is $H / 2$ for both the transmitter antenna and the receiver antenna, the propagation delay of the channel wave depends on $H, d$, and $v$.

2.3. Multipath Fading Problem. Several factors cause the attenuation and loss of channel wave propagation within a coal seam. The main factors include dielectric absorption loss due to the incomplete elasticity of the coal seam, and interface reflection loss due to channel wave reflection through the mechanical properties of the coal seam interface [22]. If $\Delta l$ represents the channel wave propagation distance in the coal seam, then the $0-1$ variable $k$ is written as follows: 
$k=\left\{\begin{array}{l}0, \text { The elastic wave does not reflect in the propagation } \Delta l, \\ 1, \text { The elastic wave has reflection in the propagation } \Delta l .\end{array}\right.$

According to the change in energy of channel wave, it is possible to obtain the following equation:

$$
\frac{E(\Delta l)-E_{0}}{E_{0}}=\eta_{\alpha} \Delta l+k \eta_{\beta}
$$

where $E(\Delta l)$ denotes the energy of the channel wave propagation distance after $\Delta l ; E_{0}$ denotes the primary energy of channel waves; and $\eta_{\alpha}$ denotes the coefficient of the absorption loss. It can be calculated using properties of the coal seam medium. $\eta_{\beta}$ denotes the interface reflection coefficient, calculated from the features of the coal seam and the rock enclosing it. The units are in $\mathrm{dB}$, and the negative symbol is used.

The absorption loss coefficient: for a clear description of the absorption level of the medium, a dimensionless quality factor $Q$ is presented [17]. This factor is defined as the ratio of storage of energy to dissipation of energy in the system. It is expressed as follows:

$$
\frac{1}{Q}=\frac{1}{2 \pi} \times \frac{\Delta E}{E}
$$

where $\Delta E$ denotes the energy lost through the unit crosssectional area within one cycle; $E$ denotes the energy that is stored as elastic energy; and $Q$ denotes the medium (coal) quality factor. This factor is an essential property of the medium.

The following equation indicates a relationship between the attenuation coefficient and the quality factor:

$$
\eta_{\alpha} \approx \frac{\omega}{2 v Q}=\frac{\pi f}{v Q} \text {. }
$$

Here, $v$ indicates the wave velocity and $f$ indicates the channel wave frequency. $\eta_{\alpha}$ denotes the coefficient of the absorption loss. Q denotes the medium (coal) quality factor.

The reflection loss coefficient: channel waves have the ability to create reflection at the interface of the coal seam. The Zoeppritz equation can be used for calculation of reflectivity. However, it is a highly complicated equation. It is assumed that channel waves are plane harmonic waves. Thus, the Zoeppritz equation can be solved in a simplified way, and the reflection coefficient can be expressed as follows:

$$
\eta_{\beta}=\frac{\rho v \cos \theta-\rho^{\prime} v^{\prime} \cos \theta^{\prime}}{\rho v \cos \theta+\rho^{\prime} v^{\prime} \cos \theta^{\prime}}
$$

where $\rho$ is the density of the coal seam; $v$ denotes the propagation velocity of the channel wave in the coal seam; $\theta$ is the incidence angle; $\rho^{\prime}$ denotes the enclosing rock density; $v^{\prime}$ denotes the channel wave propagation velocity in the enclosing rock; and $\theta^{\prime}$ denotes the angle of refraction. $\eta_{\beta}$ denotes the interface reflection coefficient. Equations (19), (21), and (22) can be solved simultaneously so that $E$ is obtained, which is expressed as follows:

$$
\begin{aligned}
E= & E_{0} e^{-\eta_{\alpha} l}+k_{1} E_{0} \eta_{\beta}=E_{0} e^{(-\pi f / v Q) l} \\
& +k_{1} E_{0} \frac{\rho v \cos \theta-\rho^{\prime} v^{\prime} \cos \theta^{\prime}}{\rho v \cos \theta+\rho^{\prime} v^{\prime} \cos \theta^{\prime}}
\end{aligned}
$$

where $k_{1}$ represents a constant; $E_{0}$ denotes the primary energy of the channel waves; and $l$ represents the channel wave propagation distance in the coal seam.

\section{Simulating Channel Wave Multipath Transmission in a Coal Seam}

A channel wave transmission was simulated according to the multipath transmission model. Table 1 gives the main parameters of the coal seam and the rock enclosing it [23].

There is a common belief that the Ricker wavelet represents the seismic wave model. Therefore, the Ricker wavelet is used as a transmitting wave with $200 \mathrm{~Hz}$ frequency. The distance between the receiver detection sensor and the transmitter transducer is $50 \mathrm{~m}$. The shape of the coal seam is rectangle with thicknesses of 6,8 , and $10 \mathrm{~m}$. The distances between the transmitter transducer and the receiver detection sensor are 3, 4, and $5 \mathrm{~m}$. COMSOL was used for running the simulation. COMSOL is a leading numerical simulation software package capable of simulating a variety of physical processes. Its simulation data provided by this software are usually utilized as a standard [24-26]. Reflected wave time delays are associated with direct waves in the model, and the simulation. Figure 2 indicates these time delays.

As observed in Figure 2, the reflected wave time delay associated with the direct wave increases with the increase in thickness of coal seam, both in the simulation and in the model. There is some discrepancy between results of the model and the simulation, as the peak of the receipt signal is used as the time of the receipt multipath signal in the simulation. There are different factors influencing the peak signal arrival time, the most significant of which is the compound nature of the channel wave signal spreading from the enclosing rock and all reflection signals. Nevertheless, the reflection signal's time delay trend shows consistency with the increase in the thickness of the coal seam.

Figure 3 indicates the signal time disparity between the multipath transmission model and COMSOL simulation.

From the comparison of the time delays obtained from the multipath transmission model and COMSOL simulation shown in Figure 3, it is found that the difference in path times is always between $-0.002 \mathrm{~s}$ and $0.002 \mathrm{~s}$. Hence, there is consistency between the model given in COMSOL and the model proposed in the current study.

The thickness of the coal seam is considered as $8 \mathrm{~m}$, and the height of the enclosing rock is $10 \mathrm{~m}$ for calculating the pressure attenuation decline of all path signals, $i$. It is calculated by the use of MATLAB and COMSOL according to the multipath transmission model and the energy loss model. It can be observed in Figure 4. 
TABLE 1: The arrangement of channels.

\begin{tabular}{lccc}
\hline Name & Density $\left(\mathrm{kg} / \mathrm{m}^{3}\right)$ & The pressure wave velocity $(\mathrm{m} / \mathrm{s})$ & The shear wave velocity $(\mathrm{m} / \mathrm{s})$ \\
\hline Coal seam & 1450 & 2200 & 1100 \\
Enclosing rock & 2600 & 3710 & 1990 \\
\hline
\end{tabular}

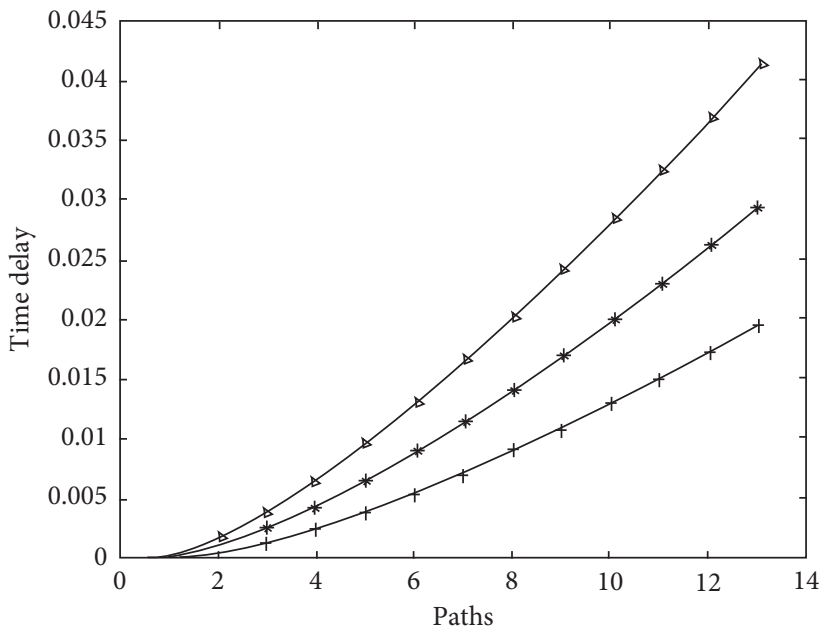

7 The height of the coal seam is $6 \mathrm{~m}$

$\rightarrow$ The height of the coal seam is $8 \mathrm{~m}$

$\rightarrow$ The height of the coal seam is $10 \mathrm{~m}$

(a)

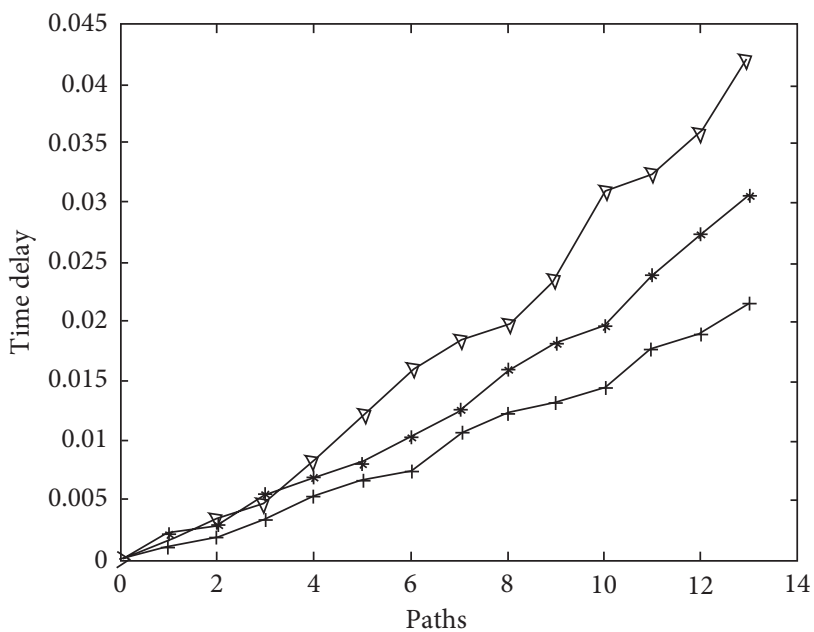

+ The height of the coal seam is $6 \mathrm{~m}$

* The height of the coal seam is $8 \mathrm{~m}$

$\rightarrow$ The height of the coal seam is $10 \mathrm{~m}$

(b)

FIGURE 2: (a) The reflected wave time delay associated with the direct wave in the model; (b) the reflected wave time delay associated with the direct wave in the simulation.

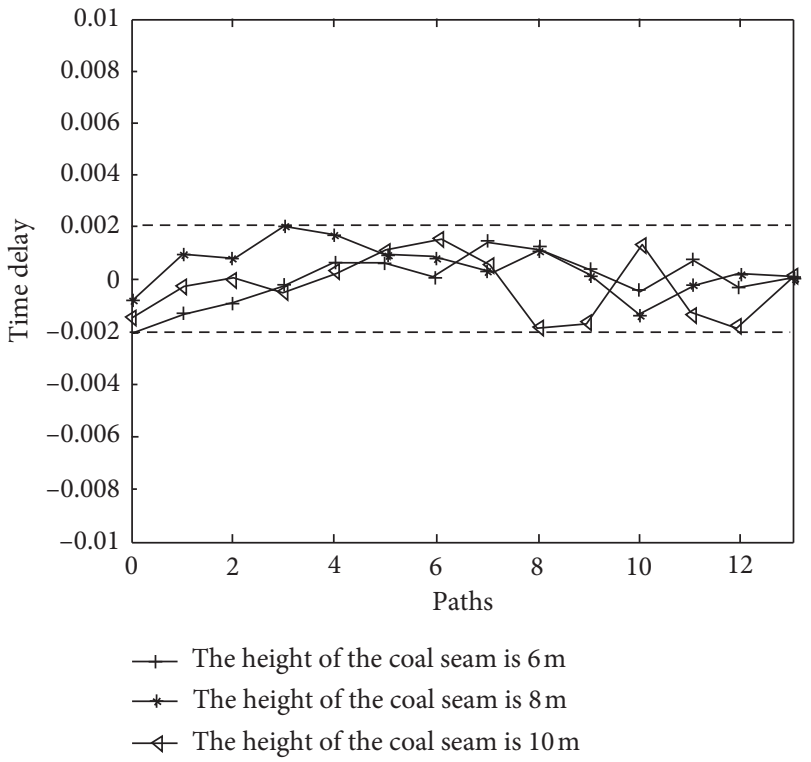

Figure 3: The signal time disparity between the multipath transmission model and COMSOL simulation.

It is observed that there is consistency between the decline tendencies of the proposed model and the COMSOL simulation results. Hence, the validity of the channel wave attenuation model in the current research is confirmed. Fluctuations are larger in the COMSOL

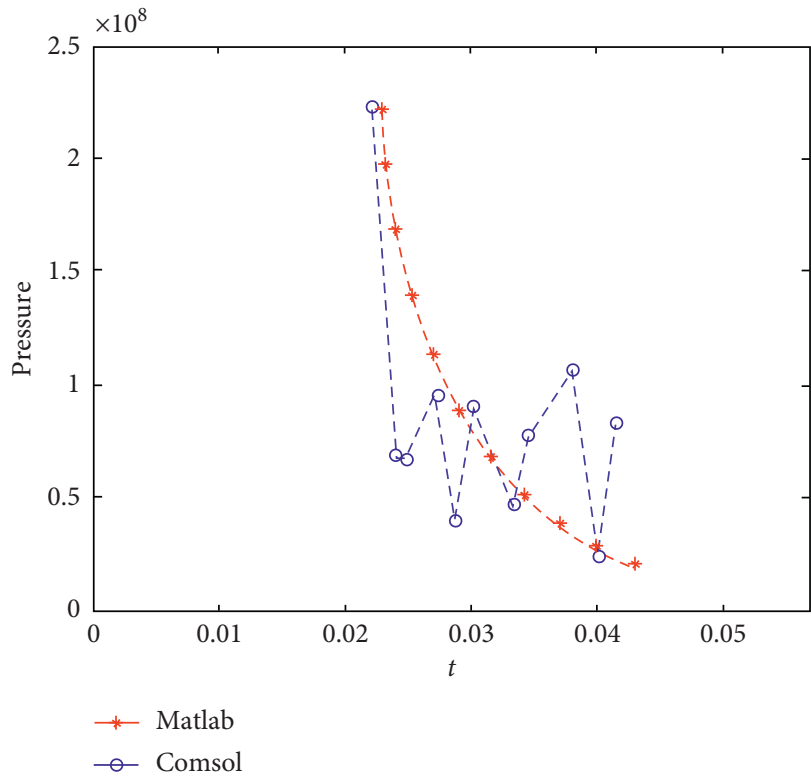

FIgURE 4: The receipt signal for an $8 \mathrm{~m}$ coal seam height.

simulation illustrated in Figure 4 due to the impact of channel waves from the enclosing rock. In addition, there is a similarity between the channel wave energy response and an attenuation following the index law over time in $50 \mathrm{~m}$. 


\section{Conclusion}

In this paper, the multipath propagation mechanism is analyzed based on simplifying the coal seam and surrounding rock, and the path difference and delay difference formulas of multipath propagation are derived. The elastic wave multipath in the formation is based on the simple experiment of the transmitter and receiver. The effect problem was mathematically modeled with MATLAB and COMSOL simulation, and the multipath fading model of a channel wave was given. It is concluded that through model simulation and comparison with COMSOL simulation results, although there are certain differences, the development trend is consistent, which verifies the correctness of the model built in this paper; the time delay of elastic wave propagation in a coal seam increases with the increase of coal seam thickness; as time increases, the elastic wave energy at a fixed distance from the source is approximately exponentially attenuated. The conclusions reached are of great significance for coal seam exploration.

\section{Data Availability}

The data used to support the findings of this study are available from the corresponding author upon request.

\section{Conflicts of Interest}

The authors declare that there are no conflicts of interest regarding the publication of this paper.

\section{Acknowledgments}

The authors wish to acknowledge the financial support of the National Natural Science Foundation of China (Grant no.61471224) and Shandong Province Key R\&D Project (Grant no.2018GHY115022). We would like to thank Editage (http://www.editage.com) for English language editing.

\section{References}

[1] Y. Le, W. Wang, Q. Shen, and D. Lyu, "Application of ISS in detection of small structures in working face," Coal Geology \& Exploration, vol. 41, no. 4, pp. 74-77, 2013.

[2] Y. Jiao, J. S. Wei, and W. W. Dou, "Application of inseam seismic exploration in fault detection," Shanxi Coal, vol. 37, no. 1, pp. 34-37, 2017.

[3] B.-L. Wang, "Automatic pickup of arrival time of channel wave based on multi-channel constraints," Applied Geophysics, vol. 15, no. 1, pp. 118-124, 2018.

[4] F. Lei, W. Wang, S. Li, X. Yao, J. Teng, and X. Gao, "Research on the channel wave field characters of goaf in coal mine and its application," in Technology and Application of Environmental and Engineering Geophysics, Q. Di, G. Xue, and J. Xia, Eds., pp. 57-70, Springer Geophysics, Singapore, 2017.

[5] N. V. Chertova and Y. V. Grinyaev, "Deformation features on the free surface and rigid interface during elastic wave reflection," Physical Mesomechanics, vol. 21, no. 3, pp. 208-215, 2018.
[6] Y. K. Ahn, H. J. Lee, and Y. Y. Kim, "Conical refraction of elastic waves by anisotropic metamaterials and application for parallel translation of elastic waves," Scientific Reports, vol. 7, no. 1, p. 12, 2017.

[7] C. Wang, X. Chen, P. Wei, and Y. Li, "Reflection and transmission of elastic waves through a couple-stress elastic slab sandwiched between two half-spaces," Acta Mechanica Sinica, vol. 33, no. 6, pp. 1022-1039, 2017.

[8] X. Zhang and S.-Y. Kung, "COD: diversity-adaptive subspace processing for multipath separation and signal recovery," Journal of VLSI Signal Processing Systems for Signal, Image and Video Technology, vol. 30, no. 1-3, pp. 235-256, 2002.

[9] T. Ioppolo and P. Rostami, "Dispersion and non-reciprocal elastic wave propagation in a membrane coupled with a uniform flow," Journal of Applied Physics, vol. 122, no. 12, 2017.

[10] X. H. Yang, L. I. De-Chun, and Y. U. Peng-Fei, "Analysis of Rayleigh channel wave dispersion in coal seam," Geophysical \& Geochemical Exploration, vol. 34, no. 6, pp. 750-752, 2010.

[11] Q. H. He, X.-Z. Chen, Z. Bin, and Y.-R. Wu, "Design of elastic wave receiving scheme in stratum communication system," Electronics Quality, vol. 38, no. 1, pp. 97-752, 2017.

[12] T. T. Nguyen and H. Oh, "A receiver for resource-constrained wireless sensor devices to remove the effect of multipath fading," IEEE Transactions on Industrial Electronics, vol. 65, no. 7, pp. 6009-6016, 2018.

[13] M. A. Akkas, "Using wireless underground sensor networks for mine and miner safety," Wireless Networks, vol. 24, no. 1, pp. 17-26, 2018.

[14] M. Matovic, S. R. Panic, Z. Popovic, M. Stefanovic, J. Zivanic, and M. Peric, "Eta-Mu modeled multipath propagation of electromagnetic waves," Technics Technologies Education Management-Ttem, vol. 7, no. 2, pp. 456-461, 2012.

[15] X. Y. Bao, D. Zhang, and S. Xie, "Study on the simulation based radio wave multipath extracting method," in Proceedings of the 2016 IEEE International Conference on Electronic Information and Communication Technology Iceict 2016, pp. 443-446, IEEE, New York, NY, USA, August 2016.

[16] H. Q. Zhang, H.-Z. Yu, P. Wang, X.-J. Gao, and M.-R. Ren, "Multipath transmission modeling and simulating of electromagnetic wave in rectangle tunnel," Chinese Journal of Radio Science, vol. 23, no. 1, pp. 195-200, 2008.

[17] J. J. Hao, Y. Zhao, and Y. J. Guo, "Power loss characteristics of plane elastic wave signal propagating in layered earth medium channel," Journal of China Coal Society, vol. 36, no. 1, pp. 211-214, 2011.

[18] S. K. Gao and L. Huang, "Research status and patent analysis in the field of transmitting communication technology," China New Telecommunications, vol. 16, no. 18, pp. 94-95, 2014.

[19] M. Baumann and M. B. van Gijzen, "Convergence and complexity study of GMRES variants for solving multi-frequency elastic wave propagation problems," Journal of Computational Science, vol. 26, pp. 285-293, 2018.

[20] P. Zhang, P. Wei, and Y. Li, "The elastic wave propagation through the finite and infinite periodic laminated structure of micropolar elasticity," Composite Structures, vol. 200, pp. 358-370, 2018.

[21] A. Dhaka, V. Bhaskar, A. Nandal, and N. Marina, "Statistical approach using phase variance for analysing correlated multipath fading environment," IET Communications, vol. 12, no. 8, pp. 948-955, 2018.

[22] J. Wang, J.-Z. Li, H. Wu, B.-L. Wang, J.-W. Hu, and G.-Z. Ji, "Tomography of transmission in-seam wave attenuation 
coefficient anddetection of collapse columns," Coal Science and Technology, vol. 43, no. 1, pp. 108-111, 2015.

[23] S. Gupta and A. Kumar, "Seismic wave attenuation characteristics of three Indian regions: a comparative study," Current Science, vol. 82, no. 4, pp. 407-413, 2002.

[24] M. B. Turgay and A. G. Yazıcıoğlu, "Numerical simulation of fluid flow and heat transfer in a trapezoidal microchannel with COMSOL multiphysics: a case study," Numerical Heat Transfer, Part A: Applications, vol. 73, no. 5, pp. 332-346, 2018.

[25] Z. Hu, J. Fan, P.-Z. Zhang, and Y.-S. Wu, "Acoustic scattering from elastic target buried in water-sand sediment," Acta Physica Sinica, vol. 65, no. 6, p. 8, 2016.

[26] N. J. Watson, T. Hazlehurst, M. J. W. Povey, A. Drennan, and P. Seaman, "COMSOL modelling of the acoustoelastic effect," Journal of Physics: Conference Series, vol. 581, Article ID 012008, 2015. 


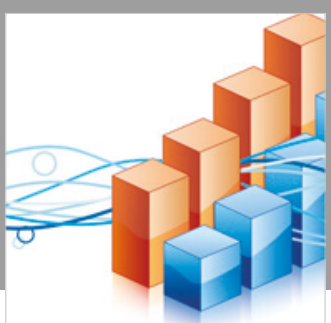

Advances in

Operations Research

\section{-n-m}
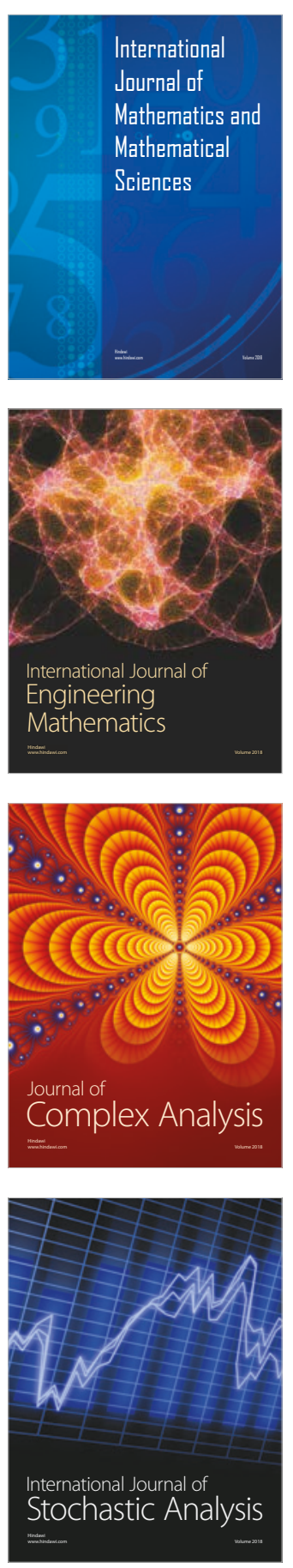
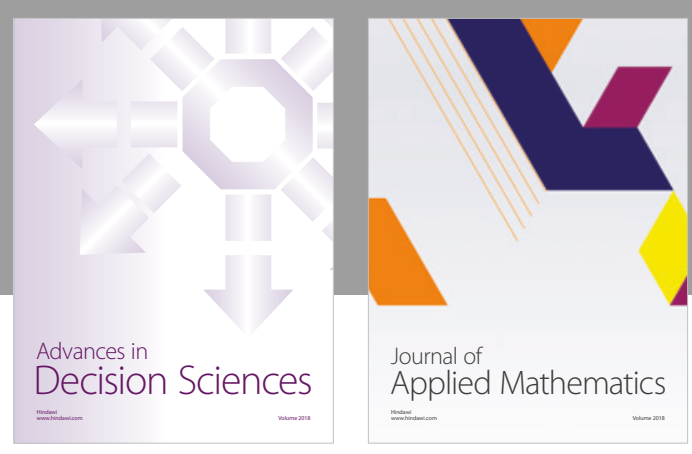

Journal of

Applied Mathematics
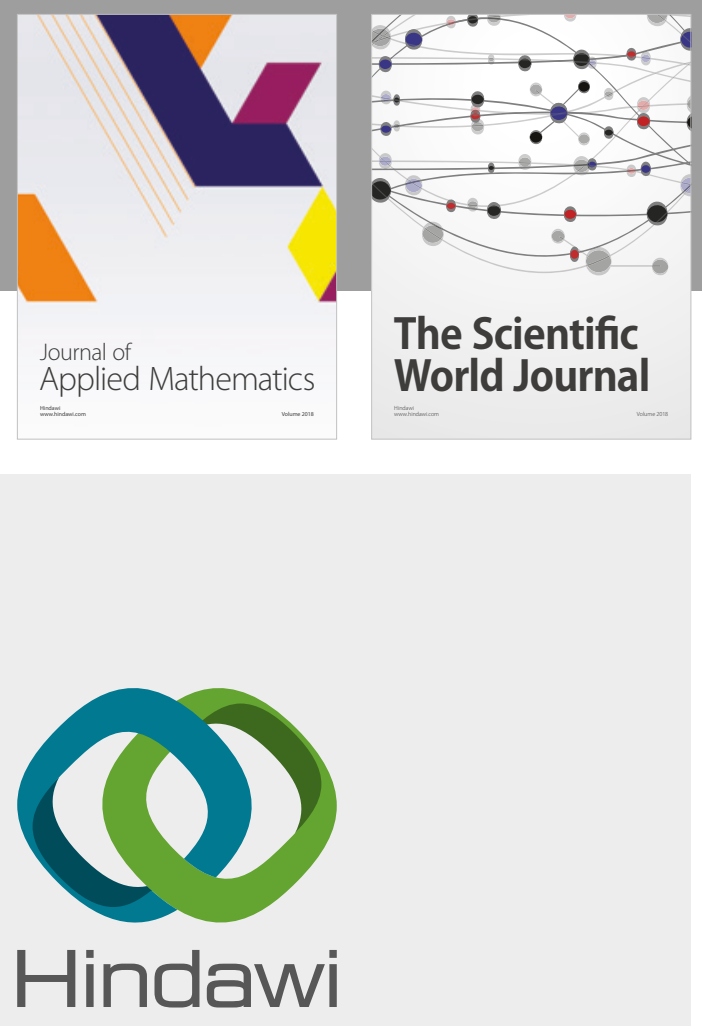

Submit your manuscripts at

www.hindawi.com

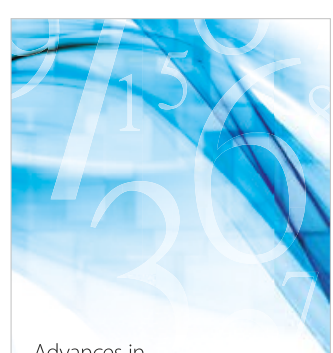

Advances in
Numerical Analysis
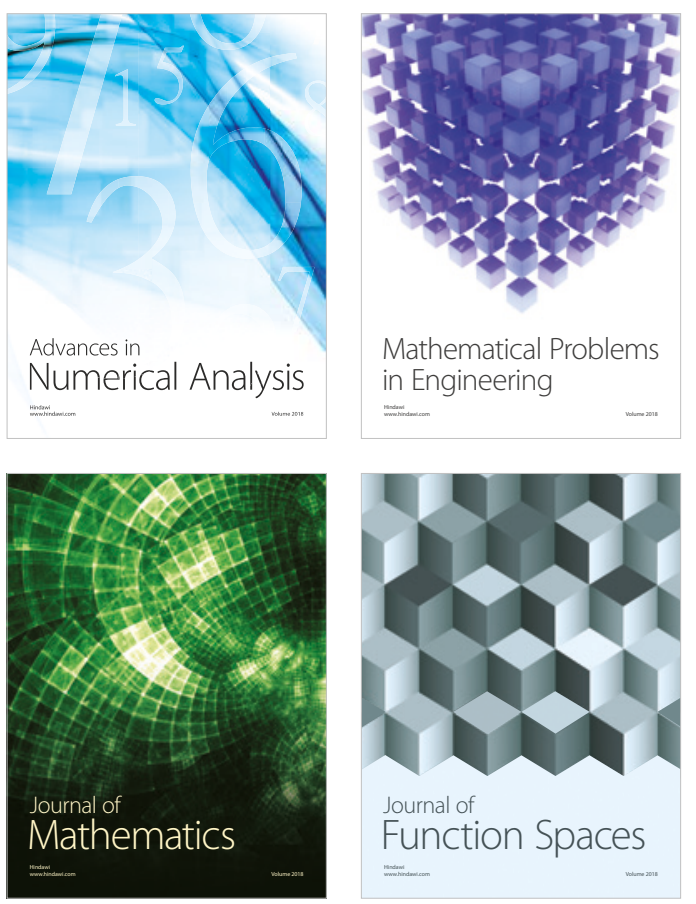

Mathematical Problems in Engineering

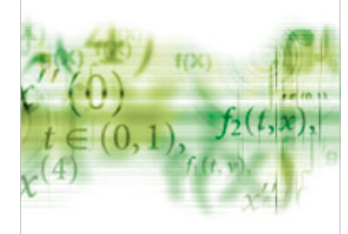

International Journal of

Differential Equations

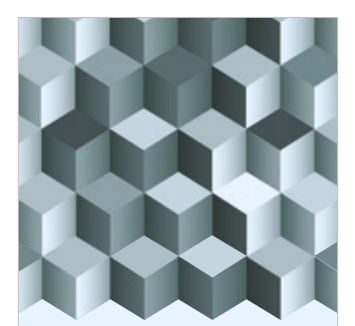

Journal of

Function Spaces

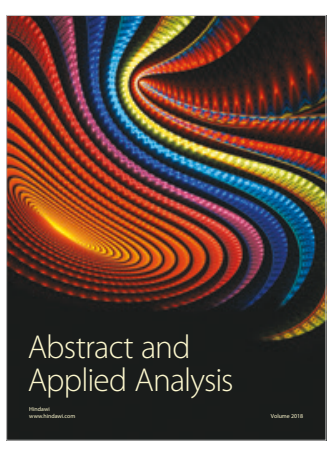

The Scientific

World Journal

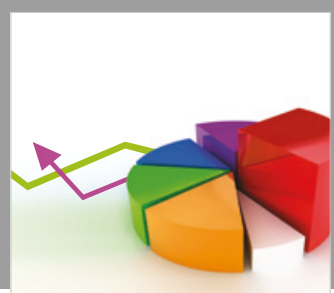

Journal of

Probability and Statistics
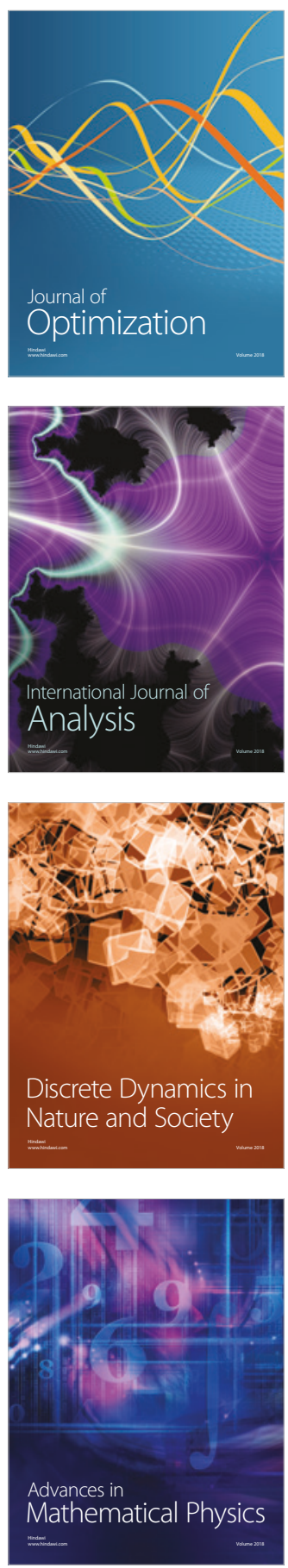